\title{
TRANSVERSE INSTABILITY DUE TO THE SPACE CHARGE DURING THE ELECTRON-COOLING BUNCHING OF ION BEAMS
}

\author{
M. Takanaka, T. Katayama, RIKEN, Wako, Japan
}

\begin{abstract}
A beam-tracking simulation has been done for study of the electron-cooling bunching of ion beams around a few hundred $\mathrm{MeV} / \mathrm{u}$. In the simulation, the field due to the space charge of a beam in a round vacuum chamber is calculated from the charge distribution of macro-particles and the image particles due to the macro-particles. The simulation results show that the bunched beams meet a transverse instability more easily than coasting beams.
\end{abstract}

\section{INTRODUCTION}

Collision experiments with ion-electron beams and ionion beams are planned at the Double Storage Ring of RIKEN RI-beam factory project. Ion-beam bunching under electron cooling has been investigated by using a beamtracking simulation for realization of the bunched beams required for the experiments. The previous-simulation results for the case of a $150-\mathrm{MeV} / \mathrm{u} \mathrm{U} \mathrm{U}_{238}^{92+}$-ion beam of 3.4 $\mathrm{mA}[1]$ have shown that 1) the beam has wide incoherenttune spreads longitudinally and transversely, 2) the beam is not trapped into resonances due to the nonlinear fields of magnets, and 3) the beam is stable longitudinally under the longitudinal broad-band impedance of $\left|Z_{L}^{b b} / n\right|=5 \Omega$ based on the broad-band impedance model.

The transverse space-charge impedance for a circle type of dipole charge distribution is described as follows;

$$
Z_{T}^{s c}=i \frac{Z_{0} R}{\beta^{2} \gamma^{2}}\left(\frac{1}{a^{2}}-\frac{1}{b^{2}}\right),
$$

where $Z_{0}$ is the free space impedance, $R$ the mean radius of a ring, $a \sqrt{2} \times$ rms beam size, and $b$ the inner radius of a round vacuum chamber. Such a type of impedance becomes no cause of instability, as the force induced through the impedance is proportional to the transverse beam position from the chamber center. There is a question whether the description is applicable to the cases of the spacecharge-dominated beams or not, or a question whether a transverse space-charge force induced through the vacuum chamber becomes a cause of instability or not. For the sake of answering the question, the field due to the space charge in a round vacuum chamber is calculated from the charge distribution of the macro-particles and the image particles due to the macro-particles in the simulation on the assumption that the longitudinal component of the field is negligible.

Besides the above calculation of the field, in the simulation where coupled-bunch phenomena are not dealt, forces induced through the transverse broad-band impedance $Z_{T}^{b b}$ and the transverse resistive wall impedance have newly been taken into account as forces acting on ions. The notation $Z_{L T}^{b b} / n$ is used just for parameterizing $Z_{T}^{b b}$;

$$
Z_{T}^{b b}=\frac{2 R}{b^{2}} \frac{Z_{L T}^{b b}}{n} .[2]
$$

The transverse broad-band impedance of $\left|Z_{L T}^{b b} / n\right|=$ $50 \Omega$ has been used on the assumption of the broad-band impedance model. The ring and the beam input parameters to the simulation have been listed in[1]. The longitudinal and transverse cooling times for a zero-current beam are 5 $\mathrm{ms}$ and about $20 \mathrm{~ms}$ in the simulation, respectively. The following simplification has been done in the simulation. 1) The vacuum chamber has a round cross section of inner radius $b$ around the ring. 2) The $\beta$ functions along the ring are equal to the average ones, and $\alpha$ functions equal to 0.3$)$ The transverse position displacement of an ion due to the momentum difference is neglected in order that the instability described later is seen to have no relation with the displacement. 4) The effects of nonlinear fields of the magnets on beams are neglected because of the authors' focus just on the instability. 5) The transverse field due to the space charge is calculated from the transverse charge distribution that is made vertically symmetry by counting half a macro-particle at the coordinates $(x, y)$ located at $(x,-y)$. Otherwise, the instability can occur in the horizontal and the vertical directions, and the analysis of the beam behavior becomes more complicated.

\section{SMALL VACUUM CHAMBER}

In the vacuum chamber of $b=40 \mathrm{~mm}$, a $150-\mathrm{MeV} / \mathrm{u} \mathrm{U}_{238}^{92+}$ ion coasting beam of $3.4 \mathrm{~mA}$ is initially electron-cooled to $6 \times \mathrm{rms}$ momentum spread of $5 \times 10^{-4}$, and is bunched by applying a fundamental RF (harmonics no., or $h=87$ ) voltage. Every RF bucket is filled with the beam. The bunch spacing is $3.0 \mathrm{~m}$. The RF voltage is increased gradually with the momentum-spread kept constant in order that the bunch length is made short.

Such a process was simulated with results as shown in Fig 1. The amplitude of the horizontal beam position becomes increased a synchrotron period after applying the RF voltage. After then, the horizontal emittance is blown up ten-times large within two synchrotron periods. The maximum instantaneous current at $5.1 \mathrm{~ms}$ is $10 \mathrm{~mA}$ at the bunch center. The horizontal phase-space distribution at $5.1 \mathrm{~ms}$ is shown in Fig 2. The instability is seen to be of the transverse dipole mode. The betatron-oscillation-phase differ- 
ence from the head to the tail of the dense bunch $1.5 \mathrm{~m}$ long is about $1 / 2 \pi$. The difference $\phi_{b}$ is understood in terms of the slippage factor $\eta$, the chromaticity $\xi$, and the bunch length $l_{b}$;

$$
\phi_{b}=\frac{\xi l_{b}}{\eta R}
$$

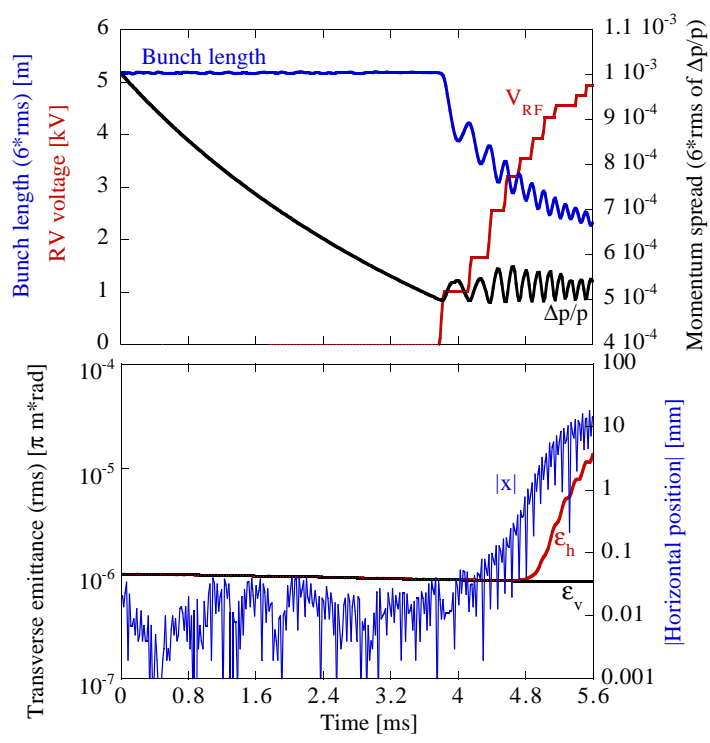

Figure 1: Instability in the vacuum chamber of $40 \mathrm{~mm}$ inner radius during the electron-cooling bunching.
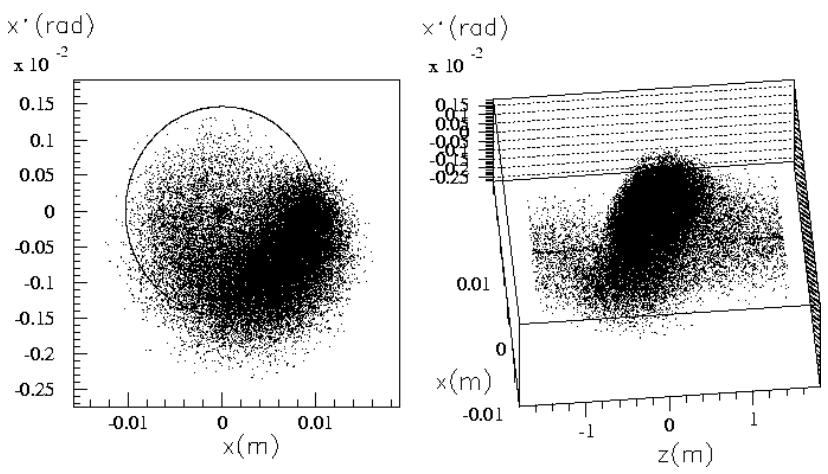

Figure 2: Horizontal phase-space distribution. The ellipse is for a reference.

\section{COHERENT TRANSVERSE OSCILLATION}

The dipole moment of a coherent transverse oscillation (transverse displacement $\times I \equiv I_{1}$ ) is Fourier-expanded as follows;

$$
I_{1}(t, s)=\sum_{\Omega, n} A(\Omega, n) \exp \left(-i \Omega t+i \frac{n s}{R}\right),
$$

where $A$ is the component amplitude, $t$ the time, $s$ the longitudinal position, $\Omega$ the coherent frequency, and the integer $n$ the oscillation number around a ring. $\omega_{0}$, described later, is the revolution frequency.
Figure 3 shows the spectrum of the horizontal dipole moment around $5.2 \mathrm{~ms}$. There is no remarkable different spectrum between the fast waves with $n>0$ and the slow waves with $n<0$. A single peak of the major structure of the signal along $\Omega / \omega_{0}-n h \approx p+0.38$ ( the integer $p=$ fixed, the integer $n=$ variable ) means that the head-tail mode of the instability is the so-called rigid-bunch mode. The signal has synchrotron satellites on the both sides, as shown in two lower of Fig.3. The fractional coherent tune is read 0.3733 and 0.3867 from peak's coordinates of the signals with $p \geq 0$ and $p<0$, respectively, while the fractional incoherent tune is estimated at $0.380 \pm 0.005$ from an FFT analysis.
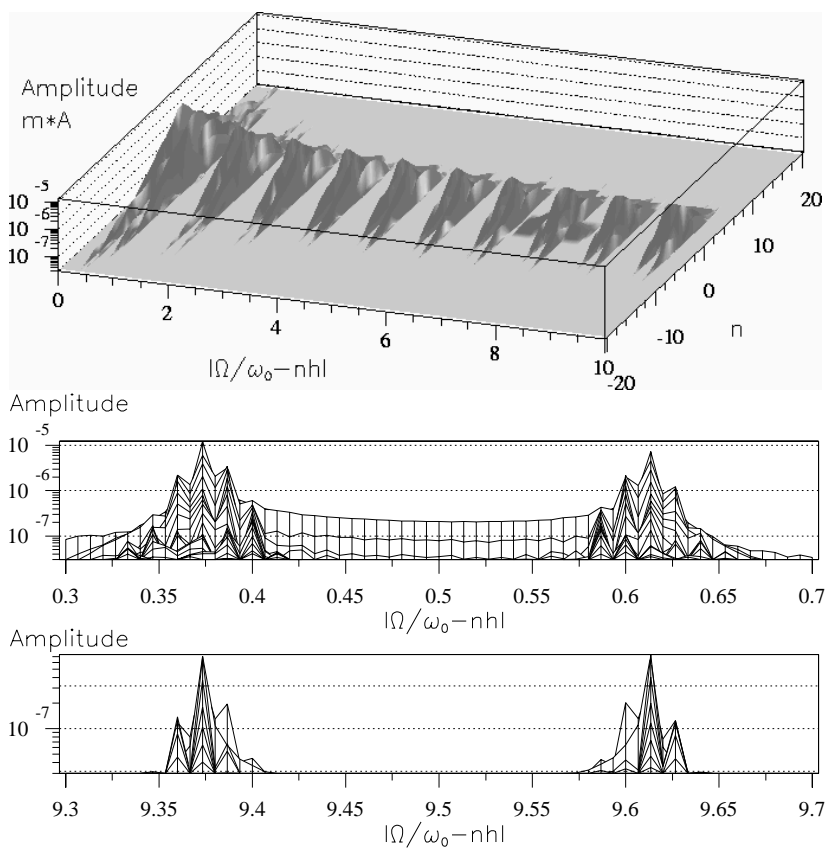

Figure 3: Spectrum of the dipole mode of coherent transverse oscillation which has low-level signals cut away.

\section{LARGE VACUUM CHAMBER}

The simulation was done on the same condition as described in Section 2 except for the double-sized vacuum chamber of $b=80 \mathrm{~mm}$. The simulation results show that the beam is as stable as in the previous case[1] up to on the way. After the beam is bunched by applying the fundamental RF voltage $20 \mathrm{kV}$ and becomes shorter than $1 \mathrm{~m}$ to the degree that the third harmonic RF voltage can be applied, the bunching is prompted by applying the third harmonic RF voltage. The beam meets the instability when the $6 \times$ rms bunch length reaches $0.6 \mathrm{~m}$. Figure 4 shows the emittance blowing-up on two impedance conditions; the one being the broad-band impedance of $\left|Z_{L T}^{b b} / n\right|=50 \Omega$ and the resistive wall impedance, and the other no broadband impedance nor the resistive wall impedance. The maximum instantaneous currents at $38.5 \mathrm{~ms}$ and $39.5 \mathrm{~ms}$ are $37 \mathrm{~mA}$ and $39 \mathrm{~mA}$ at the bunch center, respectively. The instability threshold is seen to be dependent not only 


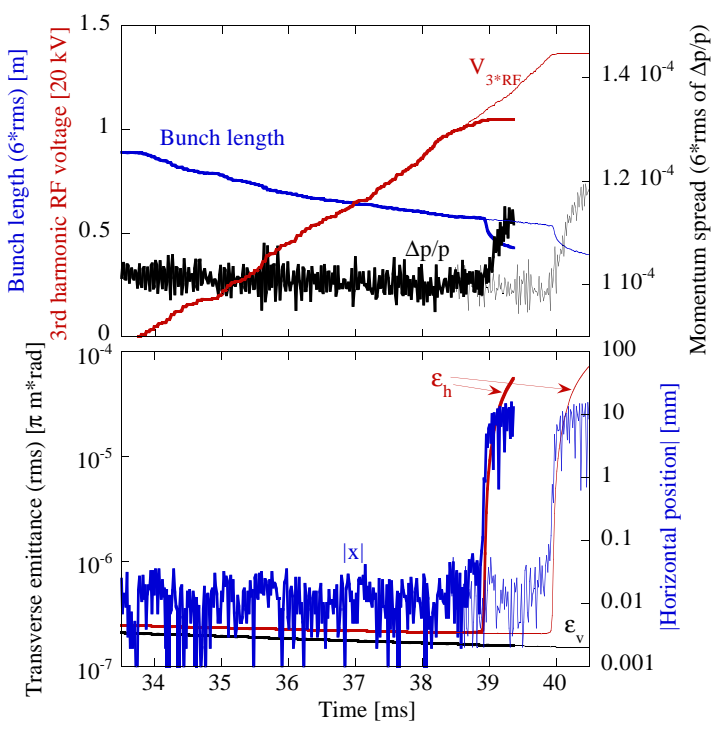

Figure 4: Instability in the vacuum chamber of inner radius $b=80 \mathrm{~mm}$ during the electron-cooling bunching. The Thick curves are for the high impedance condition, and the thin curves for no-impedance condition.

on the inner radius of the vacuum chamber but also on the transverse broad-band impedance.

It is preferable from the viewpoint of the instability cure that the inner sizes of the vacuum chamber surroundings around the ring are designed to be as wide as possible to the degree that the broad-band impedance of the ring does not increase so high.

\section{COASTING BEAMS}

For the sake of checking whether the instability described in Section 2 is merely due to the increase of the instantaneous beam current or not, the simulation was done of higher-current coasting beams under the electron cooling in the small chamber of $b=40 \mathrm{~mm}$.

The simulation results, as shown in Fig. 5, show that the coasting beam is stable even in the case where the coastingbeam current of $70 \mathrm{~mA}$ is 7 times higher than the maximum instantaneous current of the bunched beam. This means that the increase of the current is the necessary condition for the instability of the bunched beams but not the sufficient condition.

\section{COHERENCY OF TRANSVERSE OSCILLATION}

In the case of a coasting beam, even if the beam has coherent transverse oscillation on some account, when the electromagnetic interaction between the beam and the vacuum chamber surroundings are negligibly small, the coherent oscillation is diluted to disappear for the following reason. Localized ions spread around the ring and spread azimuthally $2 \pi$ on the transverse phase planes in time owing to the momentum difference.

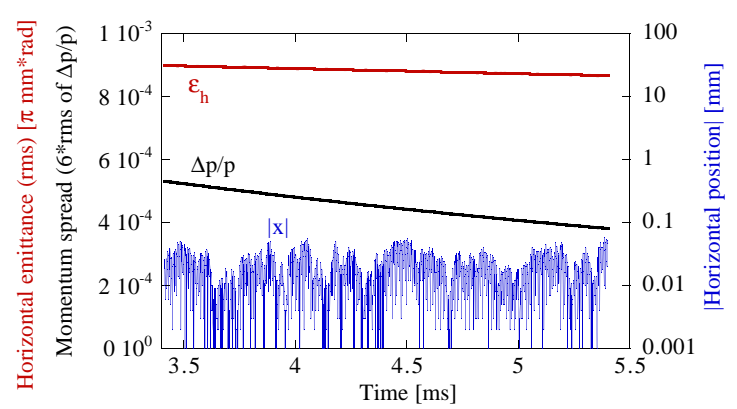

Figure 5: $70 \mathrm{~mA}$ coasting beam electron-cooled in the vacuum chamber of inner radius $b=40 \mathrm{~mm}$.

In the case of a bunched beam, the coherent transverse oscillation is not diluted for the following reason. Localized ions synchrotron-oscillate and remain localized in the RF separatrix, although ions localized near the boundary of the separatrix spread along the boundary in time owing to the non-linearity of the synchrotron frequency. There is a limitation on the betatron-oscilation-phase difference between an ion and the synchronous particle. The variation of the difference is described in the term of the longitudinal distance $z_{i}$ to the ion from the synchronous particle by Eq. (3) in which $l_{b}$ is replaced with $-z_{i}$. Therefore, the distribution of the phase difference of the beam is periodic at the synchrotron frequency.

It can be said from the above that coherency of the transverse oscillation is very low for a coasting beam and high for a bunched beam. Such high coherency is considered to be one of causes that make the bunched beams meet the instability more easily than coasting beams.

\section{CONCLUSIONS}

A beam-tracking simulation of the electron-cooling bunching of ion beams showed the following results. The spacecharge dominated beams meet a transverse instability when they are bunched by using an RF system and an electron cooler. The instability is due to the transverse field induced through the vacuum chamber by the space charge of the beam, or through the transverse space-charge impedance. The instability has the dipole mode as the transverse mode and the rigid-bunch mode as the head-tail mode. The bunched beams meet the instability more easily than coasting beams at least by 7 times in the regard of the instantaneous beam current.

Coherency of the transverse oscillation is higher for the bunched beams than for coasting beams. Such high coherency is considered to be one of causes that make bunched beams meet the instability more easily than coasting beams.

\section{REFERENCES}

[1] M. Takanaka, T. Katayama: Proc. of PAC'97, 1807(1997). M. Takanaka, T. Katayama: Proc. of EPAC'98, 1014(1998).

[2] B. Zotter, F. Sacherer: CERN 77-13, 175(1973). 